$\stackrel{N}{N=}$

Global Journals Inc.

(2)

GLOBAL JOURNAL OF MEDICAL RESEARCH: G

VETERINARY SCIENCE AND VETERINARY MEDICINE

Volume 21 Issue 2 Version 1.0 Year 2021

Type: Double Blind Peer Reviewed International Research Journal

Publisher: Global Journals

Online ISSN: 2249-4618 \& Print ISSN: 0975-5888

\title{
Characterization of Dogs and Cats Diagnosed with Diseases of the Urinary System in 2019: Veterinary Hospital, Faculty of Veterinary Medicine, University of San Carlos De Guatemala
}

By Alvarado-García, Sharon Denise, Villatoro-Chacón, Daniela Mariel

\& Arizandieta-Altán, Carmen Grizelda

Universidad de San Carlos de Guatemala

Abstract- If determined the casuistry of diseases that affect the urinary system in dogs and cats. A retrospective study was carried out at the Veterinary Hospital of the University of San Carlos de Guatemala. It was characterizing patients with diseases of the urinary system. 1,205 canine and feline medical records were evaluated in 2019. The variables breed, sex, age, pathology and geographic area were considered. The prevalence of urinary system diseases in dogs and cats in 2019 was $10.45 \%, 9.21 \%$ corresponding to dogs and $1.24 \%$ to cats. In both species, males had a higher frequency of urinary system disease, compared to females (dog $=58.56 \%$ and cat $=66.67 \%)$. The most common etiologies in dogs were lower urinary tract infections (24.65\%), bladder urolithiasis (16.90\%), and acute and chronic kidney disease (28.16\%); while in cats lower urinary tract disease (100\%) and kidney disease (5.26\%) were the main pathologies observed. In dogs, the most affected age range was in patients older than 8 years $(52.25 \%)$, while in cats it ranged from 3 to 8 years (73.34\%).

Keywords: urinary system, canine, feline, prevalence.

GJMR-G Classification: NLMC Code: WA 360

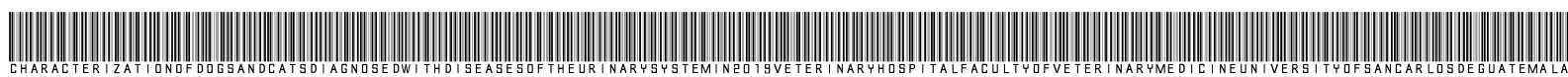

Strictly as per the compliance and regulations of:

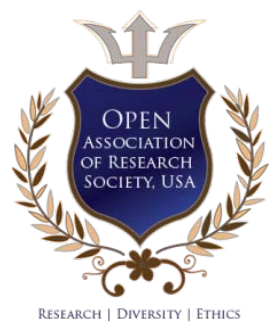

(C) 2021. Alvarado-García, Sharon Denise, Villatoro-Chacón, Daniela Mariel \& Arizandieta-Altán, Carmen Grizelda. This research/review article is distributed under the terms of the Attribution-NonCommercial-NoDerivatives 4.0 International (CC BYNC-ND 4.0). You must give appropriate credit to authors and reference this article if parts of the article are reproduced in any manner. Applicable licensing terms are at https://creativecommons.org/licenses/by-nc-nd/4.0/. 


\title{
Characterization of Dogs and Cats Diagnosed with Diseases of the Urinary System in 2019: Veterinary Hospital, Faculty of Veterinary Medicine, University of San Carlos De Guatemala
}

\author{
Caracterización De Perros Y Gatos Diagnosticados Con Enfermedades Del \\ Sistema Urinario En El Año 2019: Hospital Veterinario, Facultad De Medicina \\ Veterinaria, Universidad De San Carlos De Guatemala
}

\author{
Alvarado-García, Sharon Denise ${ }^{\alpha}$, Villatoro-Chacón, Daniela Mariel ${ }^{\sigma}$ \\ \& Arizandieta-Altán, Carmen Grizelda ${ }^{\rho}$
}

Resumen- El presente estudio tiene como finalidad determinar la casuística de enfermedades que afectan el sistema urinario en perros y gatos. Se realizó un estudio retrospectivo en el Hospital Veterinario de la Universidad de San Carlos de Guatemala caracterizando a los pacientes con enfermedades del sistema urinario. Se evaluaron 1,205 registros médicos de caninos y felinos en el año 2019. Se consideraron las variables raza, sexo, edad, patología y zona geográfica. La prevalencia de enfermedades del sistema urinario en perros y gatos en el año 2019 fue de $10.45 \%$, correspondiendo el $9.21 \%$ a perros y $1.24 \%$ a gatos. En ambas especies los machos presentaron mayor frecuencia de enfermedad del sistema urinario, respecto a las hembras (perro $=58.56 \%$ y gato $=66.67 \%$ ). Las etiologías más comunes en perros fueron las infecciones del tracto urinario bajo (24.65\%), urolitiasis vesical (16.90\%) y enfermedad renal aguda y crónica (28.16\%); mientras que en los gatos la enfermedad del tracto urinario inferior (100\%) y la enfermedad renal (5.26\%) fueron las principales patologías observadas. En los perros el rango etario más afectado fue en pacientes mayores de 8 años (52.25\%) mientras que en los gatos oscila entre 3 a 8 años (73.34\%). Las razas con mayor frecuencia fueron el French poodle (19.82\%), pacientes sin raza definida (18.92\%) y Golden Retriver (11.7\%); mientras que en los gatos en su totalidad fueron sin raza definida. Con el presente estudio se pueden observar las principales diferencias en cuanto a las etiologías que afectan a cada especie, sexo, edad y raza. Esta información permite al médico veterinario tener un panorama general del estado de salud de los pacientes, así como una línea de investigación para estudios del comportamiento epidemiológico de las patologías observadas a largo plazo y la capacitación de médicos veterinarios que aborden adecuadamente las patologías observadas.

Author a $\sigma$ p: Departamento de ayudas diagnósticas, Hospital Veterinario, Facultad de Medicina Veterinaria y Zootecnia, Universidad de San Carlos de Guatemala.

Author $\sigma:$ Autor al que se dirige la correspondencia.

e-mail:danavilla47@profesor.usac.edu.gt
Palabras clave: sistema urinario, caninos, felinos, prevalencia

Abstract-If determined the casuistry of diseases that affect the urinary system in dogs and cats. A retrospective study was carried out at the Veterinary Hospital of the University of San Carlos de Guatemala. It was characterizing patients with diseases of the urinary system. 1,205 canine and feline medical records were evaluated in 2019 . The variables breed, sex, age, pathology and geographic area were considered. The prevalence of urinary system diseases in dogs and cats in 2019 was $10.45 \%, 9.21 \%$ corresponding to dogs and $1.24 \%$ to cats. In both species, males had a higher frequency of urinary system disease, compared to females (dog $=58.56 \%$ and cat $=66.67 \%$ ). The most common etiologies in dogs were lower urinary tract infections (24.65\%), bladder urolithiasis (16.90\%), and acute and chronic kidney disease (28.16\%); while in cats lower urinary tract disease (100\%) and kidney disease $(5.26 \%)$ were the main pathologies observed. In dogs, the most affected age range was in patients older than 8 years $(52.25 \%)$, while in cats it ranged from 3 to 8 years $(73.34 \%)$. The breeds with the highest frequency were the French poodle (19.82\%), patients without a defined breed (18.92\%) and Golden Retriver (11.7\%); while in cats as a whole they were without a defined breed. With the present study, the main differences can be observed in terms of the etiologies that affect each species, sex, age and breed. This information allows the veterinarian to have a general overview of the health status of the patients, as well as a line of research for studies of the epidemiological behavior of the long-term pathologies observed and the training of veterinarians who adequately address the observed pathologies.

Keywords: urinary system, canine, feline, prevalence.

\section{INTRODUCCIÓN}

a tenencia de perros y gatos como animales de compañía ha incrementado en los últimos años. Esto debido a que son usados para llenar espacios afectivos hombre-mascota en el entorno 
familiar, guardianía, aumento de la capacidad económica de las clases sociales y el desplazamiento de familias del área rural al área metropolitana. La suma de estos factores ha influenciado a que exista un aumento en las consultas de los propietarios a los hospitales o clínicas veterinarias, para detectar anomalías en las mascotas y recibir un tratamiento adecuado (Flores, 2002). Esto a generado que las bases de datos con las que cuentan los hospitales y clínicas veterinarias sean una fuente valiosa para determinar la prevalencia de diferentes enfermedades y así predecir el comportamiento de las mismas. El realizar investigaciones de algunas enfermedades por sistemas, permite la planificación, organización e implementación de programas preventivos que son importantes para la supervivencia de los pacientes (Gonzales, 2018).

Tanto en caninos como en felinos, las enfermedades del sistema urinario, pueden aparecer en cualquier momento de vida del paciente. Es común que los propietarios de la ciudad tengan animales en espacios inadecuados para su tamaño y una alta cantidad de ellos. Esto puede ser a su vez la causa del incremento en la presentación de patologías de tipo urinario, debido a que las deposiciones diarias son controladas por los dueños, además de horarios restringidos y limitado acceso al agua, lo que se traduce en un deterioro progresivo de las funciones renales (Urbina y Campos, 2009).

Estas enfermedades son comunes, sin embargo, se encuentra escasa y anecdótica información sobre las mismas. Por lo cual se hace necesario el realizar un estudio epidemiológico que evidencie las características de la población afectada para determinar los factores de riesgo en la población estudiada. De esta manera, el médico veterinario tendrá información que facilitan su práctica clínica y brindan una mejor comprensión la presentación, fisiopatología y manejo de las enfermedades.

\section{Metodología}

El estudio se realizó en el Hospital Veterinario de la Facultad de Medicina Veterinaria y Zootecnia de la Universidad de San Carlos de Guatemala. Se realizó un estudio retrospectivo evaluando 1,205 registros médicos de los cuales 1,101 registros corresponden a perros y 104 a gatos que asistieron a consulta médica en el año 2019.

Los datos fueron clasificados según el diagnóstico clínico del médico tratante, utilizando hojas de registro. Se consideraron las variables raza, sexo, edad, patología y zona geográfica.

Para el análisis de datos se utilizó estadística descriptiva utilizando distribuciones de frecuencias para las variables. Además, se determinó la prevalencia de los pacientes con enfermedad del tracto urinario (Blair y Taylor, 2008). El software estadístico utilizado fue SPSS Statistics.

\section{Resultados}

De los 1,205 registros evaluados, la prevalencia total de enfermedades del sistema urinario en perros y gatos para el año 2019 fue de 10.45\%; correspondiendo el $9.21 \%(n=111)$ a perros y $1.24 \%(n=15)$ a gatos.

En cuanto a la procedencia de los caninos y felinos, la zona geográfica más frecuente fue de la ciudad capital 54.95\% (n=61) y $60 \% \quad(n=9)$ respectivamente. En el caso de caninos el municipio de Mixco $20.72 \% \quad(n=23)$ y Villa Nueva $13.51 \% \quad(n=15)$ fueron otras áreas frecuentes (tabla 1).

Tabla 1: Procedencia de los pacientes según área geográfica.

\begin{tabular}{ccccc}
\hline Procedencia & $\begin{array}{c}\text { Caninos } \\
\mathrm{n}=111\end{array}$ & $\begin{array}{c}\text { Felinos } \\
\mathrm{n}=15\end{array}$ & $\%$ \\
\hline Capital & 61 & 54.95 & 9 & 60 \\
Mixco & 23 & 20.72 & 2 & 13.33 \\
San Miguel Petapa & 6 & 5.41 & 3 & 20 \\
San José Pinula & 1 & 0.90 & 1 & 6.67 \\
Villa Nueva & 15 & 13.51 & & \\
Chinautla & 2 & 1.80 & & \\
Santa Catarina Pinula & 1 & 0.90 & & \\
Villa Canales & 1 & 0.90 & & \\
Livingston, Izabal & 1 & 0.90 & & \\
\hline
\end{tabular}

a) Resultados de caninos

La prevalencia en perros con enfermedad del sistema urinario fue de $10.08 \%(n=111)$. Respecto al sexo, el $41.44 \%(n=46)$ fueron hembras, mientras que el $58.56 \%(n=65)$ corresponde a los machos.
En cuanto a la raza, el French Poodle (19.82\%), pacientes sin raza definida (18.92\%) y Golden Retriver $(11.71 \%)$ fueron las más frecuentes en presentar enfermedad del tracto urinario. En otras razas se incluyen: Beagle, Bulldog inglés, Chow chow, Collie, 
Dashound, Lhasa apso, Maltes, Pastor alemán, Pastor belga, Pug, Sharpei, Yorshire terrier con 10.81\% (tabla 2).

Tabla 2: Frecuencia de enfermedades del tracto urinario en caninos por sexo y raza

\begin{tabular}{ccccccc}
\hline Raza & $\begin{array}{c}\text { Machos } \\
\mathrm{n}=65\end{array}$ & $\%$ & $\begin{array}{c}\text { Hembras } \\
\mathrm{n}=46\end{array}$ & $\%$ & $\begin{array}{c}\text { Total } \\
\mathrm{n}=111\end{array}$ & $\%$ \\
\hline French Poodle & 11 & 9.91 & 11 & 9.91 & 22 & 19.82 \\
Sin raza definida & 13 & 11.71 & 8 & 7.21 & 21 & 18.92 \\
Golden Retriever & 9 & 8.11 & 4 & 3.60 & 13 & 11.71 \\
Schnauzer & 6 & 5.41 & 4 & 3.60 & 10 & 9.01 \\
Cocker spaniel & 4 & 3.60 & 2 & 1.80 & 6 & 5.41 \\
Labrador Retriever & 2 & 1.80 & 3 & 2.70 & 5 & 4.50 \\
Husky & 3 & 2.70 & 1 & 0.90 & 4 & 3.60 \\
Chihuahua & 2 & 1.80 & 2 & 1.80 & 4 & 3.60 \\
Shih tzu & 2 & 1.80 & 2 & 1.80 & 4 & 3.60 \\
Viejo Pastor ingles & 1 & 0.90 & 1 & 0.90 & 2 & 1.80 \\
Akita & 1 & 0.90 & 1 & 0.90 & 2 & 1.80 \\
Boxer & 2 & 1.80 & 0 & 0 & 2 & 1.80 \\
Pitbull & 2 & 1.80 & 0 & 0 & 2 & 1.80 \\
Pomerania & 1 & 0.90 & 1 & 0.90 & 2 & 1.80 \\
Otras & 6 & 5.41 & 6 & 5.41 & 12 & 10.81 \\
\hline
\end{tabular}

El rango etario con mayor frecuencia de presentar enfermedad del tracto urinario fueron los pacientes mayores de 8 años (tabla 3), correspondiendo a un total del 52.25\% ( $n=58)$.

Tabla 3: Frecuencia de enfermedad del sistema urinario en caninos por rango etario y sexo

\begin{tabular}{ccccc}
\hline Rango etario & Macho & Macho & Hembra & Hembra \% \\
& $\mathrm{N}$ & $\%$ & $\mathrm{~N}$ & \\
0-12 meses & 1 & 0.9 & 2 & 1.80 \\
1-2 años & 6 & 5.41 & 3 & 2.70 \\
3-5 años & 11 & 9.91 & 10 & 9.01 \\
6-8 años & 11 & 9.91 & 9 & 8.11 \\
Mayor a 8 años & 36 & 32.43 & 22 & 19.82 \\
\hline
\end{tabular}

Respecto al diagnóstico etiológico se clasificaron las enfermedades según el rango etario, siendo los pacientes mayores de 8 años (52.25\%), los que presentaron mayores frecuencias como infecciones del tracto urinario bajo (11.27\%), urolitiasis $(7.75 \%)$ y enfermedad renal (7.04\%). En pacientes de 6 a 8 años la frecuencia de enfermedades fue de $20.42 \%$, siendo la infección del tracto urinario (4.93\%), la enfermedad renal $(4.23 \%)$ y la urolitiasis $(3.52 \%)$ las causas más frecuentes. Sin embargo, en los pacientes de 3 a 5 años la frecuencia de enfermedades del tracto urinario fue de $17.61 \%$, presentándose la infección del tracto urinario $(5.63 \%)$ y urolitiasis $(4.23 \%)$ las más frecuentes (tabla 4).

\section{b) Resultados de felinos}

La prevalencia en gatos con enfermedad del sistema urinario fue de $14.42 \%(n=15)$. Respecto al sexo, el 33.33\% $(n=5)$ fueron hembras, mientras que el $66.67 \%(n=10)$ corresponde a los machos.
Respecto a la raza en la especie felina, el $100 \%(n=15)$ de los pacientes fueron clasificados $\sin$ raza definida.

En los felinos, tanto los machos entre 1-2 años y 3-5 obtuvieran similares frecuencias $(26.67 \%, n=4)$. Por su parte, las hembras entre 6-8 años presentaron mayor frecuencia de enfermedad sistema urinario $(26.67 \%, n=4)$. En la tabla 5 se describen los rangos etarios por sexo y sus frecuencias. 
Tabla 5: Frecuencia de enfermedad del sistema urinario en gatos por rango etario y sexo.

\begin{tabular}{ccccc}
\hline Rango etario & Macho & Macho & Hembra & \multirow{2}{*}{ Hembra \% } \\
\hline 0-12 meses & $\mathrm{N}$ & $\%$ & $\mathrm{~N}$ & \\
1-2 años & 0 & 0 & 0 & 0 \\
3-5 años & 4 & 26.67 & 0 & 0 \\
6-8 años & 4 & 26.67 & 1 & 6.67 \\
Mayor a 8 años & 2 & 13.33 & 4 & 26.67 \\
& 0 & 0 & 0 & 0
\end{tabular}

En los gatos, el diagnóstico etiológico también frecuentes con las que se presentan las enfermedades fue clasificado según el rango etario, siendo los del sistema urinario (tabla 6). Los demás rangos etarios pacientes entre 1-2 años y de 6-8 las edades más obtuvieron bajas frecuencias.

Tabla 4: Frecuencia de enfermedad del sistema urinario en perros por etiología y rango etario

\begin{tabular}{|c|c|c|c|c|c|c|c|c|c|c|c|c|}
\hline & $\begin{array}{c}0-12 \text { meses } \\
n=3\end{array}$ & $\%$ & $\begin{array}{l}1-2 \\
\text { años } \\
n=11\end{array}$ & $\%$ & $\begin{array}{l}3-5 \\
\text { años } \\
\mathrm{n}=25\end{array}$ & $\%$ & $\begin{array}{l}6-8 \\
\text { años } \\
\mathrm{n}=29\end{array}$ & $\%$ & $\begin{array}{c}>8 \text { años } \\
n=74\end{array}$ & $\%$ & $\begin{array}{c}\text { Total } \\
\mathrm{n}=142^{*}\end{array}$ & $\%$ \\
\hline Infección del tracto urinario (ITU) & 1 & 0.70 & 3 & 2.11 & 8 & 5.63 & 7 & 4.93 & 16 & 11.27 & 35 & 24.65 \\
\hline Urolitiasis & & & 2 & 1.41 & 6 & 4.23 & 5 & 3.52 & 11 & 7.75 & 24 & 16.90 \\
\hline Enfermedad renal crónica (Sin estadificar) & 1 & 0.70 & 2 & 1.41 & 2 & 1.41 & 6 & 4.23 & 10 & 7.04 & 21 & 14.79 \\
\hline Lesión renal aguda & & & 1 & 0.70 & 2 & 1.41 & 3 & 2.11 & 7 & 4.93 & 13 & 9.15 \\
\hline Cistitis & & & & & 3 & 2.11 & 3 & 2.11 & 6 & 4.23 & 12 & 8.45 \\
\hline Prostatitis & & & 1 & 0.70 & 1 & 0.70 & 3 & 2.11 & 3 & 2.11 & 8 & 5.63 \\
\hline Glomerulonefritis & & & 2 & 1.41 & & & & & 4 & 2.82 & 6 & 4.23 \\
\hline Hiperplasia prostática benigna & & & & & & & 1 & 0.70 & 5 & 3.52 & 6 & 4.23 \\
\hline $\begin{array}{l}\text { Neoplasia vesical (carcinoma de células } \\
\text { transitorias) }\end{array}$ & & & & & 1 & 0.70 & & & 4 & 2.82 & 5 & 3.52 \\
\hline Enfermedad renal grado II & & & & & & & & & 4 & 2.82 & 4 & 2.82 \\
\hline Obstrucción uretral & & & & & 1 & 0.70 & & & 1 & 0.70 & 2 & 1.41 \\
\hline $\begin{array}{l}\text { Incontinencia urinaria por causa } \\
\text { neurológica }\end{array}$ & & & & & & & & & 1 & 0.70 & 1 & 0.70 \\
\hline Incontinencia urinaria idiopática & 1 & 0.70 & & & & & & & & & 1 & 0.70 \\
\hline enfermedad renal grado I & & & & & 1 & 0.70 & & & & & 1 & 0.70 \\
\hline Enfermedad renal grado III & & & & & & & & & 1 & 0.70 & 1 & 0.70 \\
\hline Quiste prostático & & & & & & & 1 & 0.70 & & & 1 & 0.70 \\
\hline Neoplasia prostática & & & & & & & & & 1 & 0.70 & 1 & 0.70 \\
\hline
\end{tabular}

*Algunos pacientes fueron diagnosticados con más de una patología

Tabla 6: Frecuencia de enfermedad del sistema urinario en gatos por etiología y rango etario.

\begin{tabular}{|c|c|c|c|c|c|c|c|c|}
\hline & $\begin{array}{l}1-2 \\
\text { años } \\
n=6\end{array}$ & $\%$ & $\begin{array}{c}3-5 \\
\text { años } \\
n=6\end{array}$ & $\%$ & $\begin{array}{l}6-8 \\
\text { años } \\
n=7\end{array}$ & $\%$ & $\begin{array}{c}\text { Total } \\
n=19^{*}\end{array}$ & $\%$ \\
\hline Cistitis idiopática & 4 & 21.05 & 4 & 21.05 & 4 & 21.05 & 12 & 63.15 \\
\hline $\begin{array}{l}\text { Neoplasia } \\
\text { vesical }\end{array}$ & & & 1 & 5.26 & 1 & 5.26 & 2 & 10.52 \\
\hline Infección urinaria & 1 & 5.26 & & & 1 & 5.26 & 2 & 10.52 \\
\hline Enfermedad renal & 1 & 5.26 & & & & & 1 & 5.26 \\
\hline Obstrucción uretral & & & 1 & 5.26 & & & 1 & 5.26 \\
\hline Urolitiasis & & & & & 1 & 5.26 & 1 & 5.26 \\
\hline
\end{tabular}

*Algunos pacientes fueron diagnosticados con más de una patología 


\section{Discusión}

Las enfermedades del tracto urinario son un conjunto de enfermedades que afectan tanto a caninos como felinos en sus diferentes estructuras: riñones, uréteres, vejiga, uretra y próstata. En este estudio, los diagnósticos presentados con mayor frecuencia en caninos son: la infección del tracto urinario, urolitiasis vesical, enfermedad renal crónica y lesión renal aguda. Según el estudio de Mendoza (2015) las principales patologías son urolitiasis, infección del tracto urinario, alteraciones de la micción, mientras que el estudio de Urbina y Campos (2009) señala que las principales son: infecciones del tracto urinario, insuficiencia renal aguda y cistitis. Estas variaciones pueden deberse a la cantidad de años estudiados, sin embargo, las infecciones del tracto urinario prevalecen como punto en común entre la presente investigación y los estudios citados.

En relación con el sexo de los caninos, se encontró que los machos presentan más patologías del tracto urinario respecto a las hembras. Estos datos son similares a los obtenidos en otros estudios como Mendoza (2015); López-Villa, et al. (2014) y Urbina y Campos (2009) en donde los más afectados fueron los machos que obtuvieron frecuencias de $74.22 \%, 71.96 \%$ y $57.51 \%$ respectivamente. Esto se debe a una asociación estadísticamente significativa de los machos a una mayor predisposición de una enfermedad del tracto urinario, por la conformación anatómica de estos, al presentar una uretra más estrecha y larga y la presencia de la próstata a diferencia de las hembras (Mendoza, 2015; Chew, 2003).

Con base a la edad, se encontró que afecta principalmente a los caninos mayores a 8 años. Urbina y Campos (2009) encontraron frecuencias similares en este rango etario. Esto se debe a un aumento en la susceptibilidad a este tipo de patologías debido a que existen diferentes factores subyacentes que alteran los mecanismos de defensa del tracto urinario o bien por un diagnóstico no temprano que desencadena problemas crónicos (Mendoza, 2015).

Respecto a la raza, se evidencio principalmente poodle, mestizos (razas no definidas), golden retriever, schnauzer, cocker spaniel como los más afectados. Datos similares se encontraron en el estudio de Mendoza (2015) que señala a las más afectadas poodle, labrador, pastor alemán, Schnauzer, cocker spaniel y chihuahua. Urbina y Campos (2009), señalan que las enfermedades del tracto urinario afectan principalmente a las razas pequeñas ( $<10 \mathrm{~kg}, 34 \%)$ lo cual se debe principalmente al menor volumen de orina producido, menor número de micciones y por consiguiente mayor concentración de minerales, bacterias, toxinas nefrotóxicas entre otros. Además, la rutina y comportamiento de los dueños al tener a las mascotas en espacios inadecuados para su tamaño, en las deposiciones controladas por los dueños y la restricción del consumo de agua, provoca el deterioro progresivo de las funciones renales (Urbina y Campos, 2009). A su vez, Dibartola (2004), señala que las razas con predisposición a presentar enfermedades del sistema renal son las pequeñas y medianas como el Schnauzer miniatura, Bichón Frise, Lasha Apso, Shi tzu, York Shire Terrier y Caniche Toy.

Respecto a la procedencia de los pacientes, la ciudad capital fue la más frecuente, seguido de diferentes municipios cercanos a la ubicación del Hospital Veterinario. Estos resultados pudieron darse, debido al bajo costo que se maneja en la institución, la diversidad de servicios y por la cercanía de los diferentes usuarios al Hospital Veterinario.

En cuanto a las etiologías encontradas, las infecciones del aparato urogenital se encuentran con mayor frecuencia en la práctica clínica de los pequeños animales. Esta puede afectar más de un órgano, localizándose en el tracto superior o en el tracto inferior. La infección de una parte del tracto urinario aumenta la posibilidad de que el resto se infecte posteriormente (Greene, 2008). Las infecciones del tracto urinario (ITU) representaron el $3.18 \%$, del total de pacientes caninos atendidos durante el periodo descrito. Se estima que aproximadamente un 10 a $14 \%$ de los caninos llevados al veterinario presentan ITU en algún momento de su vida (Senior, 2007; Westropp, 2009; Windahl, 2015).

Con respecto al sexo, se encontró que las hembras presentan con mayor frecuencia infecciones del tracto urinario con respecto a los machos. Estos datos son similares al estudio realizado por Wong et al (2015) y García et al (2019). Para esta enfermedad se reconoce al sexo como un factor predisponente, determinando que las hembras tienen un riesgo más alto a desarrollar esta enfermedad (Greene, 2008; Gaymer, 2014; Petreigne, 2017; García, 2018). La uretra de las hembras es más gruesa y corta que la uretra de los machos, lo que posiblemente hace más fácil que las bacterias asciendan hasta la vejiga, debido a que el ano de las hembras está más cerca del orificio uretral, existiendo una mayor probabilidad de contaminación fecal e inoculación de microrganismos; en ese sentido, los machos pueden tener un mecanismo protector adicional debido a las secreciones prostáticas con propiedades antimicrobianas (Elliott \& Grauer, 2007).Algunos animales pueden tener infección, tanto de la parte superior e inferior del tracto urinario, especialmente si existe insuficiencia renal (Bartges, 2004). Del mismo modo, los pacientes con insuficiencia renal crónica poseen una incidencia de un $20 \%$ en infecciones bacterianas del tracto urinario (Bartges, 2007). Esto explicaría por qué el $8.57 \%$ de los pacientes con ITU, también fueron diagnosticados con una enfermedad renal (aguda y/o crónica).

En relación con la edad, se encontró que afecta más a aquellos caninos mayores a 8 años, teniendo 
como promedio la edad de 8 años. La edad de presentación de las infecciones urinarias en perros varía desde 0,3 hasta 16 años, con una mediana de 7 años (Chew et al., 2011). Esto está asociado a que los animales de 8 años en adelante, por lo general presentan otras enfermedades concurrentes que alteran alguno de los mecanismos de defensa del tracto urinario (Mendoza, 2015; Elliot y Grauer, 2007).

En base a la raza, se han establecido algunas razas predisponentes a esta patología, encontrándose al pastor alemán, caniche miniatura y toy, labrador Retriever, Dachshund, doberman, pinsher y schnauzer miniatura con una mayor incidencia de ITU (Chew et al 2011; Petreigne 2017; Gaymer, 2014). En este caso, encontramos con mayor presentación a los caninos sin raza definida, seguido de poodle y Schnauzer. Estos datos son similares a los obtenidos por García et al (2019), en donde se presentó con mayor frecuencia en caninos mestizos, seguido de Schnauzer y cocker spaniel. Con respecto a los perros mestizos, no existen datos comparativos debido a que la mayoría incluye animales de raza (García et al, 2019).

La segunda patología con mayor frecuencia presentada fue la urolitiasis. Esta constituye la causa de aproximadamente el $18 \%$ de las consultas en las veterinarias, de caninos con afección del tracto urinario inferior (Rosas, 2021). En este caso, la incidencia de presentación de la enfermedad fue del $16.90 \%$ de los pacientes caninos que acudieron a consulta con sintomatología de estranguria, hematuria; siendo similares los resultados obtenidos por López-Villa et al (2014) de un 17.75\%.

La orina canina es una solución compleja en la que las sales pueden permanecer en solución en condiciones de sobresaturación; sin embargo, esta puede precipitarlas o formar sólidos, dando como resultado cristales, los cuales si no son expulsados se pueden unir y formar urolitos. Estos dañan el uroepitelio y provocan la inflamación del aparato urinario, predisponiendo al animal a una infección del aparato urinario (Nelson \& Couto, 2010; Mendoza, 2015).Los urolitos pueden formarse en cualquier lugar de las vías urinarias, aunque, en los perros, la gran mayoría aparece en la vejiga y pueden moverse a uretra (Baciero, 2013); solo un 5\% se localiza en riñones o en los uréteres.

Con respecto al sexo, la urolitiasis vesical, se presentó con mayor frecuencia en machos respecto a las hembras, siendo los resultados del presente estudio, similares a los obtenidos por Muralles (2021) y Del Ángel Caraza (2009). Esto se debe principalmente, a que los machos poseen una uretra más larga, estrecha y con un hueso en el pene, lo que puede impedir la salida de la orina y la precipitación de sólidos. Por lo contrario, las hembras tienen una uretra corta y amplia, lo que permite la salida de sedimento evitando la formación de urolitos o en su defecto, permite la salida de estos. (Osborne et al., 2000). Sin embargo, esto puede variar de acuerdo con el material del urolito, siendo predominante los cálculos de oxalato de calcio en el macho y los de estruvita en la hembra, que se asocian principalmente a infecciones del tracto urinario (Bermúdez, 2017).

En cuanto a la edad, se observó que afecta principalmente a los caninos mayores a 8 años. En el estudio realizado por Muralles (2021), hace referencia que el rango de edad para esta afección se encuentra entre los 5 a 12 años principalmente. Esto puede deberse a los cambios morfológicos y funcionales que ocurren en los animales con el paso del tiempo, o bien por diferentes medicamentos que predisponen a esta patología (Lekcharoensuk et al., 2002; Osborne et al., 2000 y Del Ángel Caraza, 2009).

Con relación a la raza, se encontró que los caninos de raza pequeña (62.5\%, schnauzer, chihuahua, maltes, yorshire, pomerania, poodle, entre otros) son más susceptibles en comparación a las razas de talla grande (37.5\%, golden retriever, viejo pastor inglés, husky, otros). Esto se debe principalmente, a la proporción en el consumo de agua, menor volumen de orina, menor número de micciones y, por tanto, mayor concentración de minerales. (Stevenson, 2002; Rosas, 2021).

La enfermedad renal fue la tercera causa más frecuente de enfermedad del tracto urinario. Esta se clasificó en lesión renal aguda (9.15\%), enfermedad renal crónica estadio I (0.70\%), enfermedad renal crónica estadio II (2.82\%), enfermedad renal crónica estadio III (0.70\%) y enfermedad renal crónica sin estadificar (14.79\%). En clínica de pequeños animales, la casuística ante pacientes renales señala que la Enfermedad Renal Crónica (ERC) es la patología renal más frecuente; ocurre cuando los mecanismos de compensación renal no son capaces de mantener las funciones propias del riñón: regulación de electrolitos, del equilibrio hídrico y ácido base y síntesis de hormonas (Chew, 2011); con prevalencias mundiales entre el $0.5 \%$ - $1.5 \%$ en caninos (Brown, 2007; González, 2018 y Miguel, Gimenez, Meder, 2021).

Del total de caninos diagnosticados con enfermedad renal crónica, solo el 22.22\% fue clasificada en los diferentes estadíos: el grado I fue diagnosticado $0.7 \%$ en caninos de $3-5$ años; grado II, en 4 caninos mayores a 8 años (2.82\%) y en grado III, $0.7 \%$ en mayores de 8 años. Los pacientes que no fue posible estadificar por falta de pruebas de laboratorio y seguimiento se presentaron en el rango etario a partir de los 6 años en adelante (14.79\%). La International Renal Interest Society, IRIS, sugiere la clasificaciónde los enfermos renales crónicos en base a sus niveles de creatinina plasmática en cuatro niveles, que a su vez se pueden sub clasificar en función de la proteinuria y de la presión arterial. Este tipo de clasificación permite 
unificar criterios para el diagnóstico, pronóstico y tratamiento de estos pacientes.

Con respecto al sexo, no se encontró ninguna diferencia entre hembras y machos respecto a la enfermedad renal. Según Adams (2004), no existe una predilección sexual en la presentación de IRC en perros ni gatos. Estos resultados son similares al estudio de Häfelin, (2008), Chandler, (2007), en todos estos estudios la proporción de machos y de hembras era muy cercana a 1:1.

En base a la edad, se observó con mayor frecuencia en pacientes mayores de 8 años. En el estudio realizado por González (2018), reporta que se observa principalmente en caninos mayores a 10 años (45\%). Esto se debe principalmente por la persistencia de alguna enfermedad renal que no fue diagnosticada a temprana edad y que, con el paso del tiempo, se presentan las alteraciones en las nefronas del canino. En un adulto o geriátrico, existe una limitante ante la capacidad del organismo de producir nefronas y compensar las dañadas, lo que hace que el problema sea persistente en el tiempo y de curso progresivo (Valdés, 2002; González, 2018). Por lo general, las edades avanzadas son las más afectadas, se presentan en un $10 \%$ en pacientes geriátricos; sin embargo, esta patología se puede presentar en animales de cualquier edad (Brown, 2007; González, 2018; Cortadellas, 2010). En el caso de los caninos que presentaron la enfermedad a una edad temprana, se debe al diagnóstico temprano y a la metodología utilizada (Watson et al., 2003). Se encontró que el $0.70 \%$ de los caninos menores a un año, diagnosticados con enfermedad crónica. Se ha reportado que en animales jóvenes está relacionado con problemas congénitos y/o familiares debido a la presencia y persistencia de glomérulos fetales o inmaduros, engrosamiento de la membrana basal glomerular o un rasgo presente en un grupo de perros emparentados; mientras que la adquirida, ocurre en mayor frecuencia en animales seniles (Cavalera, et al, 2021; Polzin, 2011; Chandler et al., 2007; Gough \& Thomas, 2004; Birchard \& Sherding, 1996).

Con relación a la raza, se encontró que afectó principalmente a caninos de raza definida (80\%), los cuales incluye al Poodle, Golden Retriever, Cocker Spaniel, Schnauzer. La ERC está descritaen muchas razas caninas y puede ocurrir en algunos mestizos, cursando a edad temprana (menor a 5 años), y en razas como Alaskan Malamute, Beagle, Boxer, Montañés de Berna, Bull Terrier, Chow Chow, Cocker spaniel, Golden Retriever, Lhasa Apso, Poodle estándar, Shih tzu, Schnauzer miniatura, Rottweiler, Samoyedo y Shar pei. (DiBartola, 2002; Chandler et al., 2007; González, 2015; Häfelin, 2008).

La lesión renal aguda es un síndrome potencialmente reversible que se produce como consecuencia de una rápida alteración de la función renal por una enfermedad que cursa en horas a días. (Nelson \& Couto, 2010). Dicha alteración da lugar a un aumento rápido y progresivo de los metabolitos tóxicos en sangre (azotemia) junto con desbalances hidroelectrolíticos y acido-base, que se reflejan en una falla para concentrar la orina y oliguria en la mayoría de los casos (Daza et al., 2008)

Con respecto al sexo, el $69.23 \%$ de los caninos fue diagnosticado con lesión renal aguda, fueron machos. Similares datos, se obtuvieron en los estudios realizados por Häfelin (2008); Vaden (1995) y Behrend (1996). Lo cual podría estar relacionado a la presentación de origen renal. Ya que como se observó anteriormente, la presentación de urolitiasis en machos fue de $79.17 \%$, siendo esta una de las causas más comunes de la lesión renal aguda post renal (Cowgill y Elliott, 2002; Häfelin, 2008).

En relación con la edad, se presentó principalmente en animales mayores de 8 años, datos parecidos al estudio de Häfelin (2008); Behrend (1996). Según Cowgill (2004), esta enfermedad puede ocurrir a cualquier edad, sin embargo, existe mayor predisposición en animales seniles debido a procesos morfológicos y funcionales.

Con respecto a la raza, se encontró que afectó principalmente a animales de raza pura siendo estos Golden retriever $30.77 \%$ y Husky Siberiano $15.38 \%$.Sin embargo, para esta patología no existe una predilección racial, cualquier canino puede verse afectado por diversos factores (Cowgill, 2004; Vaden, 1995). En el estudio realizado por Häfelin (2008), hubo una predominancia de esta patología en raza poodle y cocker spaniel, sin embargo, aclaran que esto puede deberse a una mayor presencia de estas razas en la casuística global del hospital.

Con respecto a felinos, las patologías urinarias diagnosticadas fueron: Enfermedad del tracto urinario inferior (FLUTD por sus siglas en inglés) y enfermedad renal. El FLUTD incluye las siguientes etiologías: cistitis idiopática, infección urinaria, neoplasia vesical, obstrucción uretral y urolitiasis. Estos hallazgos son similares a los reportados por otros autores como Forrester y Roudebush (2007), Suárez, Bertolani, Avellaneda y Tabar (2013), Osborne et al (1996), con la diferencia que reportan urolitiasis y tapones uretrales como la segunda causa de FLUTD, en un rango de 10 a $21 \%$ de presentación.

La enfermedad del tracto urinario inferior (FLUTD), es una alteración de la vejiga urinaria y/o uretra de los gatos, afectando a todas las edades, siendo cada vez más visible en felinos que viven en interiores (Hostutler et al., 2005). Del total de pacientes felinos atendidos en el Hospital Veterinario (104), el $14.42 \%$ fue diagnosticado con FLUTD. Sin embargo, se reporta que la incidencia anual correspondiente a esta patología es de un 1\% (Suárez, et al 2013; Cely, 2016). Este aumento, puede deberse a diferentes factores 
ambientales, de comportamiento y alimentación a los que son sometidos los felinos (Westropp, 2008; Suárez, Bertolani, Avellaneda, Tabar 2013).Según Drobatz y Costello (2012), Sævik, Trangerud, Ottesen, Sørum y Eggertsdóttir (2011), García y Barcena (2014) y Westropp (2008) la principal causa de FLUTD, es la cistitis idiopática, afectando a dos tercios de los gatos. Como se pudo observar en el presente estudio.El diagnóstico de FLUD se realiza por exclusión (Harvey y Tasker, 2014; Cortadellas, 2010; Forrester y Roudebush, 2007, Kim et al 2017; Forrester, 2015, Chew, 2011). No se conoce la patogénesis de este diagnóstico, sin embargo, está asociado a estrés y al comportamiento que tienen los felinos a lo largo de su vida; siendo las principales, la baja ingesta de agua y el alto consumo de comida solida (concentrado), lo que originan una disminución de los niveles de glucosaminoglicanos, y como consecuencia una reducción del efecto protector del epitelio urinario, lo que ocasiona que la orina penetre y origine una inflamación en la vejiga urinaria (Baciero, 2011; Westropp, 2008; Houston \& Elliott, 2010). En el caso de estos felinos, el $73.33 \%$ tienen una dieta basada en concentrado y solo el $26.67 \%$ de ellos, adicionalmente, se les proporciona comida húmeda razón por la cual, podría presentarse con mayor frecuencia esta enfermedad.

Suárez, et al (2013), refieren que los felinos más susceptibles son aquellos con sobrepeso, poca actividad física, limitado acceso al exterior, alimentados con dieta seca y que conviven con más gatos. Sin embargo, Cely (2016), hace la observación, que no en todos los casos se aplican estos factores de riesgo y se deben de tener siempre en cuenta la anamnesis del paciente.

Con respecto a las infecciones urinarias, menos del $2 \%$ de los felinos con FLUTD, tienen como causa esta patología (Nelson \& Couto, 2010). Normalmente, el tracto urinario del gato es un ambiente hostil para el crecimiento bacteriano y estas suelen presentarse cuando los animales pierden los mecanismos de defensa. Por lo general se dan, cuando hay una migración ascendente de las bacterias por la uretra a la vejiga (Bartges, 2004; Litster et al., 2009). Según Chew et al., (2011) entre un 0,1 a $1 \%$ de los gatos presenta ITU durante su vida.

Con respecto al sexo, se presentó con mayor frecuencia en machos (66.67\%) en comparación con hembras (33.33\%). Estos datos son similares a los obtenidos en los estudios realizados por Urbina y Mosquera (2009), Astuty, Tjahajati y Nugroho (2020) en donde la mayor presentación de enfermedades del tracto urinario bajo corresponde a machos $(61.1 \%$ y $69.9 \%$ respectivamente). Esta diferencia con respecto al género es significativa en el caso de FLUTD (Dorsch, et al 2014), ya que se debe especialmente a una diferencia anatómica en el largo de la uretra; siendo los machos los más afectados ya que esta, es bastante larga y presenta dos zonas de estrechamiento (Saevik et al, 2011) en comparación con las hembras (Bengoa, 1995 \& Cely, 2016); además, existe un factor de riesgo, al realizarles una castración, se disminuye el crecimiento de la uretra, originando esta enfermedad (Buffington et al., 2006; Cortadellas, 2010; Suárez, Bertolani Avellaneda \& Tabar 2013).

En cuanto a la edad, la frecuencia de presentación fue muy amplia, 1-8 años, afectando a temprana edad principalmente a los machos y luego a las hembras. Según Urbina \& Campos, 2009, la mayor frecuencia de presentación de patologías urinarias se encuentra en felinos mayores a 8 años (33.1\%), luego de 2 a 5 años (24.8\%), y por último de 5 a 8 años (20.9\%). Sin embargo, las patologías del tracto urinario pueden presentarse a cualquier edad, siendo las causas idiopáticas más frecuentes en animales jóvenes; mientras que, en los animales seniles son aquellas patologías relacionadas con la disminución de las defensas (García \& Barcena, 2014; Astuty, Tjahajati y Nugroho, 2020)

En relación con la raza, el 100\% de los felinos con enfermedad del tracto urinario, no tenía una raza definida. Urbina \& Campos (2009), mencionan que se presenta en $56.1 \%$ en felinos sin raza definida, y el 43.9\% corresponde a felinos de raza, dentro de los que se encuentra: siamés, persa, angora. Los resultados obtenidos, pueden deberse, al mayor porcentaje de felinos mestizos que asisten a consulta en comparación con felinos de razas puras.

\section{Referencias Bibliográficas}

1. Adams L. Chronic renal failure. In: Tilley, L.P.; Smith, F.W. The 5 Minutes Veterinary Consult. $3^{a}$ edición. Baltimore, Estados Unidos: Lippincott, Williams \& Wilkins; 2004. p.1124-1125

2. Astuty ATJE, Tjahajati I, Nugroho WS. Detection of feline idiopathic cystitis as the cause of feline lower urinary tract disease in Sleman Regency, Indonesia. Vet World. 2020 Jun; 13(6):1108-1112. https://doi. org/10.14202/vetworld.2020.1108-1112

3. Baciero $\mathrm{G}$. Enfermedades del tracto urinario inferior en el gato. AMVAC. 2011 [agosto 2021] No. 32 Comunicación científica Royal Canin Ibérica, S.A. Madrid: España Disponible en: http://axonveterinaria.net/web_axoncomunicacion/auxiliarvete rinario/32/AV_32_Revista_completa.pdf

4. Baciero G. Urolitiasis caninas. AMVAC. 2013 [agosto 2021 ] No. 43 Disponible en: http://axonveterinaria.net/web_axoncomunicacion/auxiliarveteri nario/43/AV_43_Revista_completa.pdf

5. Bartges J. Diagnosis of urinary tract infection. Vet Clin N Am Small Anim Pract. 2004; 34(4): 923933. 
6. Bartges J. Bacterial urinary tract infection. In: The North American Veterinary Conference. United States, Tennessee. University of Tennessee. 2007. p. 671- 673.

7. Behrend E, Grauer G, Mani I, Groman R, Salman M. Hospital-acquired acute renal failure in dogs: 29 cases (1983-1992). J Am Vet Med Assoc. 1996. 208(4): 537-541.

8. Bengoa A. Cristaluria felina. Cuantificación de glicoproteínas urinarias bajo diferentes condiciones de alimentación. [Tesis doctoral]. Madrid, España: Universidad Complutense de Madrid: 1995. Recuperado de https://proxy.europeana.eu/ 9200101/BibliographicResource_1000126624580?vi ew $=$ http\%3A\%2F\%2Fwww.ucm.es\%2FBUCM\%2Fte sis\%2F19911996\%2FD\%2F2\%2FAD2010501.pdf\&di sposition=inline\&api_url=https\%3A\%2F\%2Fapi.eur opeana.eu\%2Fapi

9. Bermudez M. Urolitiasis canina. [Tesis de pregrado]. Caldas, Antioquia: Corporación Universitaria Lasallista Facultad de Ciencias Administrativas y Agropecuarias Medicina Veterinaria; 2017. Recuperado de http://repository. lasallista.edu.co/dspace/bitstream/10567/2145/1/Ur olitiasis_canina.pdf

10. Birchard SJ, Sherding RG. Manual Clínico de Pequeñas Especies. México: McGraw Hill; 1996

11. Blair C, Taylor R. Bioestadística. México: Pearson Prentice Hall. 2008. 556 p.

12. Brown SA. Management of chronic kidney disease. In Elliott J, Grauer G. Manual of Canine and Feline Nephrology and Urology. Gloucester, Inglaterra: British Small Animal Veterinary Association; 2007. 223-230

13. Buffington T, Westropp JL, Chew DJ, Bolus RR. Risk factors associated with clinical signs of lower urinary tract disease in indoor-housed cats. Rev. JAVMA. 2006; 228 (5), 722-725.

14. 14. Cavalera MA, Gernone F, Uva A, D'Ippolito P, Roura X, Zatelli A. Clinical and Histopathological Features of Renal Maldevelopment in Boxer Dogs: A Retrospective Case Series (1999-2018). Animals. 2021, 11, 810. https://www.mdpi.com/2076-2615/ 11/3/810/htm

15. Chandler M, Elwood C, Murphy K, Gajnayake I, Syme H. Juvenile nephropathy in 37 boxer dogs. J Small Anim Pract. 2007; 48: 690-694. https://online library.wiley.com/doi/10.1111/j.1748-5827.2007.004 $01 . x$

16. Chew D. Manual de nefrología y urología en los pequeños animales. Barcelona, España: Editorial Salvat S.A; 2003 p. 358

17. Chew D, Dibartola S, Schenck P. Cystitis and Urethritis: Urinary Tract Infection. In: Canine and Feline Nephrology and Urology. $2^{\mathrm{a}}$ ed. Missouri,
United States: Elsevier Saunders. 2011. p. 240-268; p306-340.

18. Cely Niño DM. Reporte de caso clínico: enfermedad del trato urinario inferior felino (FLUTD). [Tesis de pregrado]. Bogotá, Colombia: Universidad de Ciencias Aplicadas y Ambientales; 2016. Recuperado de: https://repository.udca.edu.co/ bitstream/handle/11158/585/articulo\%20repor;jsessi onid $=8$ E9115221E458B49703CDFA4750E7CE8?se quence $=1$

19. Cortadellas O. Estadificación y manejo de la enfermedad renal crónica. Manual de nefrología y urología canina y felina. España: Ed. Servet; 2010. p.161.

20. Cowgill L, Elliott D. Falla renal aguda. In: Ettinger, S. y Feldman, E. Tratado de Medicina Interna Veterinaria. 5 ta ed. Buenos Aires, Argentina: Intermédica.; 2002. 1798-1819 p.

21. Cowgill L. Acute renal failure. In: Tilley, L.P.; Smith, F.W. The 5 Minutes Veterinary Consult. $3^{a}$ ed. Baltimore, Estados Unidos: Lippincott, Williams \& Wilkins; 2004. 1122-1123 p.

22. Daza González MA, García Pérez E, Fragio Arnold C. Manejo de urgencias en la insuficiencia renal aguda. AMVAC. 2008; No. 26, 12-21. Disponible en: http://axonveterinaria.net/web_axon-comuni-cacion/ centroveterinario/26/cv 26 Insuficiencia renal.pdf

23. Del Ángel Caraza J. Estudio epidemiōógico de la urolitiasis canina en México [Tesis de pregrado]. México: Universidad de León; 2009. Recuperado de: https://dialnet.unirioja.es/servlet/tesis?codigo= 108071

24. Dibartola, S. Enfermedad renal familiar en caninos y felinos. In: Ettinger, S. y Feldman, E. Tratado de Medicina Interna Veterinaria. 5ta ed. Buenos Aires, Argentina: Intermédica; 2002. 1891-1896 p.

25. Dorsch R, Hartmann K, Sauter-Louis C. Feline lower urinary track disease in a German cat population: A retrospective analysis of demographic data. Causes and clinical signs. Tierarztl. Prax. Ausg. K. Kleintiere Heimtiere; 2014 42(4): 231-239.

26. Drobatz KJ, Costello MF. Emergencias en medicina felina. Buenos aires, Argentina: Intermedica; 2012.

27. Elliott J, Grauer G. Manual of canine and feline nephrology and urology. $2^{a}$ ed. Dorset, Inglaterra: British Small Animal Veterinary Association (BSAVA); 2007. $298 p$

28. Forrester S, Roudebush P. Evidence-Based Management of Feline Lower Urinary Tract Disease. Vet Clin Small Anim. 2007; 37: 533-558 p.

29. Forrester SD, Towell TL. Feline idiopathic cystitis. Vet. Clin. North Am. Small Anim. Pract. 2015; 45(5): 783-806 p.

30. Flores Cuellar HR. Las características del servicio al cliente que generan lealtad hacia las tiendas de mascotas [Tesis de pregrado]. Guatemala: 
Universidad Rafael Landívar Facultad De Ciencias Económicas y Empresariales; 2002. Recuperado de: $\quad$ http://biblio3.url.edu.gt/Tesis/lote01/FloresHector.pdf

31. García, L.M.; Barcena, M. Hospital Veterinario Molins: Principales patologías del tracto urinario inferior felino. [internet]. [Consultado agosto 2021]. Disponible en: https://www.portalveterinaria.com/ articoli/articulos/24655/principales-patologias-deltracto-urinario-inferior-felino.html

32. García SM. Estudio retrospectivo de los aislados bacterianos y su sensibilidad antimicrobiana en caninos con diagnóstico de infección del tracto urinario atendidos en la Clínica de Animales Menores de la FMV-UNMSM entre los años 20122017 [Tesis de pregrado] Lima: Perú: Universidad del Perú; 2018. Recuperado de https://cybertesis. unmsm.edu.pe/handle/20.500.12672/10090

33. García M Milagros, Díaz C Diego, Huerta M Carlos, Olazábal L Juan, Barrios-Arpi Manuel, Chipayo G Ysaac. Análisis retrospectivo de agentes bacterianos y patrones de susceptibilidad antibiótica en casos de infecciones del tracto urinario en caninos domésticos (2012-2017). Rev. investig. vet. Perú: 2019; No. 30(4): 1837-1844 p. Disponible en: http://www.scielo.org.pe/ scielo.php? script=sci_arttext\&pid = S1609-91172019000400049 \&lng=es. $\quad$ http://dx.doi.org/10.15381/rivep.v30i4. 17263.

34. Gaymer EC. Descripción de registros clínicos de perros y gatos con infecciones del tracto urinario (ITU) [Tesis de pregrado]. Santiago, Chile: Universidad de Chile Facultad de Ciencias Veterinarias y Pecuarias Escuela de Ciencias Veterinarias; 2014. Recuperado de: http://repositorio.uchile.cl/bitstream/handle/2250/131689/Descri pci\%c3\%b3n-de-registros-cl\%c3\%adnicos-deperros-y-gatos-con-infecciones-del-tracto-urinario\%28ITU\%29.pdf?sequence=1 \&isAllowed=y

35. González JF. Relación de las patologías caninas más frecuentes que se presentan en la clínica de pequeños animales en la zona noroeste de la Comunidad de Madrid, con las variables edad, raza, sexo y tamaño. [Tesis doctoral]. Madrid, España: Universidad Complutense de Madrid; 2015. Recuperado de: https://eprints.ucm.es/id/ eprint/33266/

36. González-Castillo LF, Sanmiguel-Plazas RA. Acercamiento a la enfermedad renal crónica en caninos y felinos geriátricos. Ciencia y Agricultura. 2018; 15(2): 71-81. Disponible en: https:// repositorio.uptc.edu.co/bitstream/001/2294/1/PPS 938_Acercamiento_enfermedad_renal_canina.pdf

37. Gough A, Thomas A. Breed predispositions to disease in dogs and cats. Oxford, UK: Blackwell Publishing Ltd.; 2004225 p.
38. Greene C. Enfermedades infecciosas del perro y el gato. $3^{\circ}$ ed. Buenos Aires, Argentina: Intermedica; 2008 Cap 91 p.1027-1046

39. Häfelin R. Estudio descriptivo de registros clínicos de pacientes caninos y felinos con diagnóstico de insuficiencia renal. [Tesis de pregrado] Santiago de Chile: Universidad de chile Facultad de Ciencias Veterinarias y Pecuarias Escuela de Ciencias Veterinarias; 2008. Recuperado de: http:// repositorio.uchile.cl/bitstream/handle/2250/133639/ Estudio-descriptivo-de-registros-cl\%C3\%ADnicosde-pacientes-o-descriptivo-de-registros-cl\%C3\%A Dnicos-de-pacientes-\%20renal.pdf?sequence $=1$

40. Harvey A, Tasker S. Manual de medicina felina. Barcelona, España: Ediciones S; 2014

41. Hostutler RA, Chew DJ, DiBartola SP. Recent Concepts in Feline Lower Urinary Tract Disease. Rev. Vet Clin Small Anim. 2005; No. 35: 147-170 p.

42. Houston DM, Elliott DA. Tratamiento nutricional de las patologías del tracto urinario inferior en el gato. En: Pibot P, Biourge V, Elliott D, Flatin JC. Enciclopedia de la nutrición clínica felina. París: Royal Canin; 2010. p 284-321.

43. IRIS, International renal interest society (2019). Disponible en: http://www.iris-kidney.com/

44. Kim Y, Kim H, Pfeiffer D, Brodbelt D. Epidemiological study of feline idiopathic cystitis in Seoul, South Korea. J. Feline Med. Surg. 2017; 20(10): 913-921p.

45. Lekcharoensuk C, Osborne CA, Lulich J P, Pusoonthornthum R, Kirk CA, Ulrich LK, Koehler LA, Carpenter KA, Swanson LL. Associations between dietary factors in canned food and formation of calcium oxalate uroliths in dogs. Am $J$ Vet Res. 2002; 63(2): 163-169. Disponible en: https://doi.org/ 10.2460/ajvr.2002.63.163.

46. Litster A, Thompson M, Moss S, Trott D. Feline bacterial urinary tract infections: An update on an evolving clinical problem. Vet J. 2009; 187(1): 18- 22.

47. López-Villa J, Mendoza López C, Del Ángel Caraza J, Quijano Hernández IA, Barbosa Mireles MA. Patologías que afectan al tracto urinario caudal de los perros y gatos. Hospital Veterinario para Pequeñas Especies FMVZ-UAEMex. Toluca, México: 2014. [Consultado agosto 2021] Disponible en: http://ri.uaemex.mx/bitstream/handle/20.500. 11799/58002/5\%c2\%ba\%20Simposio_HILLS_2015. 1-12.pdf?sequence $=3 \&$ isAllowed $=y$

48. Mendoza Cl. Enfermedades del tracto urinario caudal de los perros. [Tesis de pregrado]. México: Universidad Autónoma del Estado de México; 2015. Recuperado de: http://ri.uaemex.mx/handle/20.500. 11799/58760

49. Miguel M, Gimenez M, Meder A. Neutrophil gelatinase-associated lipocalin (NGAL): Biomarker of acute kidney injury in dogs. Brazilian Journal of 
Animal and Environmental Research. 2021; No.4. 2490-2503 p. Disponible en: https://www.brazilian journals.com/index.php/BJAER/article/view/31236

50. Muralles RA. Caracterización clínica y epidemiológica de pacientes caninos con urolitiasis en el Hospital Veterinario de la Facultad de Medicina Veterinaria y Zootecnia en el periodo de febrero 2015-noviembre 2017 [Tesis de pregrado]. Guatemala: Universidad de San Carlos de Guatemala; 2021 Recuperado de: http://www. repositorio.usac.edu.gt/15899/1/Tesis\%20Med.\%20 Vet.\%20Rogelio\%20Augusto\%20Muralles\%200scal \%20actualizacion.pdf

51. Nelson RW, Couto CG. Small Animal Internal Medicine. España: ELSEVIER; 2010

52. Osborne CA, Kruger JM, Lulich JP. Fe line lower urinary tract disorders; definition of term and concept. Vet. Clin. North Am. Small Anim. Pract. 1996; 26(2): 169-179 p.

53. Osborne CA, Bartges JW, Lulich JP, Polzin DJ, Allen TA. Urolitiasis canina. En: Hand MS, Thatcher CD, Remillard RL, Roudebush P, editores. Nutrición clínica en pequeños animales. 4th ed. Bogotá: Panamericana; 2000. p. 711-733.

54. Petreigne C. Diagnóstico de infección urinaria en canino macho. [Tesis de pregrado]. Tandil, Argentina: Universidad Nacional del Centro de La Provincia de Buenos Aires; 2017. Recuperado de https://www.ridaa.unicen.edu.ar/xmlui/bitstream/han dle/123456789/1322/Petreigne\%2C\%20Celia.pdf?se quence $=1$ \&isAllowed $=y$

55. Polzin DJ. Chronic kidney disease. Nephrology and urology of small animals. Blackwell publishing. 2011; 433-471.

56. Rosas Martínez A. Reporte de urolitiasis vesical en un canino en la Clínica Veterinaria UNIPAZ. Rev CITECSA. 2021; 12(19), 33-43. Recuperado de: https://revistas.unipaz.edu.co/index.php/revcitecsa/ article/view/223

57. Saevik BK, Trangerud C, Ottesen N, Sorum H, Eggertsdottir AV. Causes of lower urinary tract disease in Norwegian cats. J. Feline Med. Surg. 2011; 13(6): 410-417 p.

58. Senior D. Management of urinary tract infections. In: BSAVA Manual of Canine and Feline Nephrology and Urology. $2^{a}$ ed. London, U.K.: British Small Animal Veterinary Association (BSAVA); 2007. pp. 282-288.

59. Stevenson AE. The incidence of urolithiasis in cats and dogs and the influence of diet in formation and prevention of recurrence. [Tesis doctoral]. Londres: University College London; 2002. Recuperado de: http://www.ivis.org/advances/rc_es/A4309.

0608.ES.pdf? $\llcorner A=2$.

60. Suárez M, Bertolani C, Avellana A, Tabar M. Las vías urinarias "Tan sencillas como complejas": Cistitis intersticial "esas locas vejigas". Formación continuada, asociación de veterinarios españoles especialistas en pequeños animales (AVEPA). 2013. P. 31-37 Disponible en: https://avepa.org/pdf/ proceedings/URINARIO_PROCEEDING2013.pdf

61. Urbina Bohórquez EM, Campos Mosquera C. Estudio retrospectivo de la prevalencia de enfermedades del sistema urinario en una población de caninos y felinos en un lapso de 15 años (1993 - 2008) en la ciudad de Bogotá, Colombia. [Tesis de pregrado]. Bogotá, Colombia: Universidad de la Salle; 2009. Recuperado de: https://ciencia.lasalle.edu.co/medicina_veterinaria/2 91

62. Vaden $\mathrm{S}$, Levine $\mathrm{J}$, Correa $\mathrm{M}$, Jameson $\mathrm{P}$, Williams L, Stumpf N, Breitschwerdt E. Retrospective analysis of 106 dogs with acute renal failure. J Vet Intern Med. 1995; 9:209.

63. Valdés A. Actualización en el manejo de la insuficiencia renal crónica. En: VI Curso Internacional de Medicina y Cirugía en Pequeños Animales. Quintero, Chile. 2002; 125-133.

64. Watson D, Lefebvre $H$, German A, Font A. Diagnóstico Precoz de la Insuficiencia Renal Crónica. Aniwa Publishing. París, Francia. 2003; 19$24 \mathrm{pp}$.

65. Westropp, J. Feline Idiopathic Cystitis: Pathophysiology and Management. World Small Animal Veterinary Association World Congress Proceedings. Estados Unidos: University of California. 2008. Disponible en: https://www.vin. com/apputil/content/defaultadv1.aspx?id=3866633 \&pid $=11268 \&$

66. Westropp J. Diagnosis and Management of Bacterial Urinary Tract Infections in Dogs and Cats. In: 4th International Baytril ${ }^{\circledR}$ Symposium. Florence, Italy. 18-19 June 2009. Bayer HealthCare, Animal Health. p. 16-23.

67. Windahl U. Bacterial infections in dogs with special reference to urinary tract infections, surgical site infections and Methicillin-resistant Staphylococcus pseudintermedius. 2015; 90p. Disponible en: https://pub.epsilon.slu.se/12200/1/windahl u 15050 8.pdf

68. Wong C, Epstein S, Westropp J. Antimicrobial susceptibility patterns in urinary tract infections in dogs (2010-2013). J Vet Intern Med. 2015; (29): 1045-1052. 